\section{INTEGRAL INEQUALITY ESTIMATES FOR P.D.E.S IN UNBOUNDED DOMAINS}

\section{J. N. Flavin}

\section{Introduction}

In the context of problems involving P.D.E.s (boundary value problems, initial boundary value problems etc.), inequality estimates for certain, non-negative, $L^{2}$ integral measures of the solution are of interest: typically, they yield, inter alia, uniqueness of solution and continuous dependence upon data. The purpose of this note is to show how such estimates for weighted measures may be obtained in the context of unbounded domains where (prescribed) growth of the solution at infinity is allowed. Three results are proved which represent perhaps the simplest cases of such; they are believed to be new, or, at least, not well known.

In the matter of notation, subscripts denote partial differeniliation, and it will be convenient occasionally to write (for the weight function

$$
g(\xi)=e^{-\lambda \xi}
$$

where $\lambda$ is a constant, $\xi$ being the appropriate independent vari-

\section{An Hyperbolic Equation}

Let us commence with (arguably) the simplest example of the (linear) wave equation in an unbounded region, where growth at infinity is allowed which is not faster than exponential. A weighted energy inequality is derived therefor which has obvious analogues in all the common cases of wave-like equations in unbounded media (of whatever type); moreover, it has analogues in nonlinear elastodynamics and electrodynamics for certain classes of constitutive

equations. It is an easy matter to modify the proof of the proposition given hereunder in order to include a source term (as in subsequent Propositions).

Consider $C^{2}$ solutions of the initial boundary value problem: $u(x, t)$ satisfies

$$
\begin{aligned}
& u_{t t}=u_{x x}, 0<x<\infty, t>0, \\
& u(x, 0), u_{t}(x, 0) \text { specified }, 0<x<\infty, \\
& u(0, t)=0, \\
& u_{x}, u_{t}=O\left(e^{\mu x}\right) \text { as } x \rightarrow \infty
\end{aligned}
$$

where $\mu$ is a given positive constant (here and subsequently).

Proposition 1. The weighted energy associated with the foregoing, namely

$$
E(t)=\int_{0}^{\infty} e^{-\lambda x} \frac{1}{2}\left(u_{t}^{2}+u_{x}^{2}\right) d x
$$

where $\lambda$ is a constant such that

$$
\lambda>2 \mu,
$$

satisfies the inequality

$$
E(t) \leq E(0) \exp (\lambda t)
$$

Proof: Differentiation, use of (1), followed by integration by parts using (3)-(6) yields

$$
\frac{d E}{d t}=\int_{0}^{\infty} g(x)\left(u_{t} u_{x}\right)_{x} d x=\lambda \int_{0}^{\infty} g(x) u_{x} u_{t} d x
$$

Use of the arithmetic-geometric inequality gives

$$
\frac{d E}{d t} \leq \lambda E
$$

and the required estimate (7) follows on integration. 
Remark 1. Uniqueness of solution, and continuous dependence (in a certain sense) on data, for the problem (1)-(4) follow by standard means.

Remark 2. The inequality (7) is sharp in the sense that

$$
E(t) /\{E(0) \exp (\lambda t)\} \sim 1 \text { as } 2 \mu / \lambda \uparrow 1
$$

when

$$
u(x, t)=\sinh \mu x e^{\mu t}
$$

Remark 3. An estimate for $u(x, t)$, in terms of the initial data, follows from (7) via. Schwarz's inequality:

$$
\begin{aligned}
u^{2}(x, t)=\left(\int_{0}^{x} u_{x} d x\right)^{2} & \leq\left(\int_{0}^{x} e^{\lambda x} d x\right)\left(\int_{0}^{x} e^{-\lambda x} u_{x}^{2} d x\right) \\
& \leq 2 \lambda^{-1}\left(e^{\lambda x}-1\right) E(t)
\end{aligned}
$$

\section{A Parabolic Equation}

Consider smooth solutions of the I.B.V.P. for the heat equation (with source) in a semi-infinite rod: $u(x, t)$ satisfies

$$
\begin{aligned}
& u_{t}=u_{x x}+f(x), 0<x<\infty, t>0 \\
& u(x, 0)=\text { specified, } \\
& u(0, t)=0 \\
& u, u_{x}, u_{t}=O\left(e^{\mu x}\right) \text { as } x \rightarrow \infty
\end{aligned}
$$

where $\mu$ is a positive constant, and $f$ is a given function such that

$$
f=O\left(e^{\mu x}\right) \text { as } x \rightarrow \infty \text {. }
$$

Proposition 2. The weighted $L^{2}$ measure of solution associated with the foregoing, namely

$$
F(t)=\int_{0}^{\infty} e^{-\lambda x} u^{2} d x
$$

where $\lambda$ is a constant such that

$$
\lambda>2 \mu
$$

satisfies

$$
F(t) \leq\left[\left\{F^{1 / 2}(0)+\sigma\right\} e^{\lambda^{2} t / 4}-\sigma\right]^{2},
$$

where

$$
\sigma=4 S \lambda^{-2}
$$

with

$$
S=\left\{\int_{0}^{\infty} g(x) f^{2} d x\right\}^{\frac{1}{2}}
$$

Proof: Differentiation, use of (8), integration by parts (twice) using (10), (11), (13), (14) yields

$$
\begin{aligned}
\frac{d F}{d t} & =-2 \int_{0}^{\infty} g(x) u_{x}^{2} d x+\lambda \int_{0}^{\infty} g(x)\left(u^{2}\right)_{x} d x+2 \int_{0}^{\infty} g(x) u f d x \\
& =-2 \int_{0}^{\infty} g(x) u_{x}^{2} d x+\lambda^{2} F+2 \int_{0}^{\infty} g(x) u f d x
\end{aligned}
$$

Applying the inequality of Appendix 1 to the first term, and Schwarz's inequality to the last, we obtain

$$
\frac{d F}{d t} \leq \frac{1}{2} \lambda^{2} F+2 S F^{1 / 2},
$$

whence the proposition follows by straightforward integration $\left(F^{1 / 2}=\sqrt{F}\right)$.

Remark 4. Similar to Remark 1.

Remark 5. The inequality (15) is sharp in the sense that both sides are asymptotically equivalent as $2 \mu / \lambda \uparrow 1$ when $u$ has the form

$$
u(x, t)=U(t) \sinh \mu x
$$

(the associated $f(x), u(x, 0)$ also being proportional to $\sinh \mu x$ ). Remark 6. A similar-though more involved-analysis may be carried out, mutatis mutandis, for the measure

$$
F(t)=\int_{0}^{\infty} e^{-\lambda x} u_{x}^{2} d x .
$$


Moreover, one may deduce therefrom pointwise bounds, in terms of data, for $|u(x, t)|$ (cf. Remark 3).

\section{An Elliptic Equation}

Consider smooth solutions of Poisson's equation in a semi-infinite strip: $u(x, y)$ satisfies

$$
\begin{aligned}
& u_{x x}+u_{y y}=f(x, y), 0<x<1,0<y<\infty, \\
& u(x, 0)=0, \\
& u(0, y), u(1, y) \text { specified, } \\
& u, u_{x}, u_{y}=O\left(e^{\mu y}\right) \text { as } y \rightarrow \infty,
\end{aligned}
$$

where $\mu$ is a given positive constant, and $f(x, y)$ is a given function such that

$$
f(x, y)=O\left(e^{\mu y}\right) \text { as } y \rightarrow \infty .
$$

Proposition 3. The weighted $L^{2}$ (cross-sectional) measure of solution of the foregoing, namely

$$
F(x)=\int_{0}^{\infty} e^{-\lambda y} u^{2} d y
$$

where $\lambda$ is a constant such that

$$
\lambda>2 \mu
$$

satisfies the estimate

$$
F^{1 / 2}(x) \leq G(x)
$$

where $G(x)$ satisfies the (simple) boundary value problem

$$
G^{\prime \prime}+\left(\frac{\lambda^{2}}{4}\right) G=-\phi(x)
$$

wherein

$$
\phi(x)=\left\{\int_{0}^{\infty} e^{-\lambda y} f^{2}(x, y) d y\right\}^{1 / 2}
$$

subject to

$$
G(0)=F^{1 / 2}(0), \quad G(1)=F^{1 / 2}(1),
$$

(both of which are available from data) PROVIDED

$$
\lambda<2 \pi \text {. }
$$

Proof: Successive differentiations, use of (20) and integration by parts using $(21),(23),(25),(26)$, together with the inequality in Appendix 1, yield

$$
\begin{aligned}
F^{\prime}(x) & =\int_{0}^{\infty} 2 g(y) u u_{x} d y, \\
F^{\prime \prime}(x) & \geq 2 \int_{0}^{\infty} g u_{x}^{2} d y-\frac{\lambda^{2}}{2} F+\int_{0}^{\infty} 2 g f u d x .
\end{aligned}
$$

Assume that $F>0$ strictly. Noting that

$$
\left(F^{1 / 2}\right)^{\prime \prime}=\frac{1}{2} F^{-3 / 2}\left[F F^{\prime \prime}-\frac{1}{2} F^{\prime 2}\right]
$$

(25), (26), (32), (33), together with Schwarz's inequality (used twice) lead to

$$
\left(F^{1 / 2}\right)^{\prime \prime}+\left(\frac{\lambda^{2}}{4}\right) F^{1 / 2} \geq-\phi(x)
$$

The proof is completed on invoking Appendix 2 with $h$ identified with $F^{1 / 2}-G$. The restriction that $F>0$ strictly may be removed without difficulty, but this is not pursued here.

Remark 7. The restriction (31) is irremovable as the example $u=\sinh \pi y \sin \pi x$ shows.

Remark 8. It is verifiable that the inequality (27) is sharp in the sense that both sides are asymptotically equivalent as $2 \mu / \lambda \uparrow 1$ when $u$ has the form

$$
u=U(x) \sinh \mu y
$$


(the associated $f(x, y), u(0, y), u(1, y)$ being suitable constant multiples of $\sinh \mu y$ ).

Inequality estimates for integral measures of P.D.E.s in many contexts are treated in [1]. They include ones for unbounded regions which allow (prescribed) growth at infinity, of which the ones given here are perhaps the simplest examples: one of the earliest - perhaps even the earliest - examples of these latter techniques in mechanics occur in [2] and in the papers cited therein.

We conclude with the remark that where estimates for unbounded media of the type considered in this paper are concerned, the exponential weight function used throughout is by no means essential: growth conditions other than the exponential ones together with complementary weight functions, can equally well be contemplated.

\section{References}

[1] J. N. Flavin and S. Rionero, Qualitative Estimates for Partial Differential Equations, anl Introduction. CRC Press: Florida, 1995.

[2] G. P. Galdi and S. Rionero, Weighted Energy Methods in Fluid Dynamics and Elasticity. Lect. Notes in Math. 1134. Springer-Verlag: 1985.

\section{Appendix 1}

Proposition. If $\Phi(x) \in C^{1}(0 \leq x \leq \infty)$ is such that $\Phi(0)=0$ and

$$
\int_{0}^{\infty} e^{-\lambda x}\left(\Phi_{x}^{2}+\Phi^{2}\right) d x<\infty
$$

for some positive constant $\lambda$, but is otherwise arbitrary, then

$$
\frac{\lambda^{2}}{4} \int_{0}^{\infty} e^{-\lambda x} \Phi^{2} d x \leq \int_{0}^{\infty} e^{-\lambda x} \Phi_{x}^{2} d x .
$$

Proof: The hypotheses imply that

$$
\int_{0}^{\infty}\left(e^{-\lambda x} \Phi^{2}\right)_{x} d x=0
$$

whence

$$
\begin{aligned}
\left(\frac{\lambda^{2}}{4}\right)\left(\int_{0}^{\infty} e^{-\lambda x} \Phi^{2} d x\right)^{2} & =\left(\int_{0}^{\infty} e^{-\lambda x} \Phi \Phi_{x} d x\right)^{2} \\
& \leq \int_{0}^{\infty} e^{-\lambda x} \Phi^{2} d x \int_{0}^{\infty} e^{-\lambda x} \Phi_{x}^{2} d x
\end{aligned}
$$

by Schwarz's inequality. (For a proof under weaker hypotheses, see [2].)

The inequality above is sharp in the sense that both sides are asymptotically equivalent when

$$
\Phi=\sinh \mu x,
$$

$\mu$ being a constant such that $\lambda>2 \mu$, and $2 \mu / \lambda \uparrow 1$.

\section{Appendix 2}

Proposition. Suppose that $h(x) \in C(0 \leq x \leq 1)$ satisfies

$$
\begin{aligned}
& \left.h^{\prime \prime}+\Lambda h \geq 0, \quad x \in\right) 0, \mathbf{1}(, \\
& h(0)=h(1)=0,
\end{aligned}
$$

where $\Lambda$ is any positive constant such that $\Lambda<\pi^{2}$, then

$$
h \leq 0 \text {. }
$$

The case $\Lambda=0$ is geometrically obvious (curve under chord property for convex functions). Different proofs of the proposition may be found in $[1 \mathrm{~A}]$ by means of maximum principles, and in [2A] by means of Wirtinger's inequality.

\section{References (A)}

[1A] M. H. Protter and H. Weinberger, Maximum-Principles in Differential Equations. Prentice-Hall: New York, 1967.

[2A] E. F. Beckenbach and R. A. Bellman, Inequalities. Springer-Verlag: 1965 .

J. N. Flavin,

Department of Mathematical Physics,

University College,

Galway,

Ireland. 